\title{
Second Law Analysis of Forced Convective Cooling in a Channel with a Heated Wall Mounted Obstacle
}

\author{
Z. Kheirandish, S. A. Gandjalikhan Nassab ${ }^{*}$, M. Vakilian \\ Mechanical Engineering Department, Shahid Bahonar University, Kerman, Iran \\ Email: *Ganj110@uk.ac.ir
}

Received May 18, 2013; revised June 18, 2013; accepted June 25, 2013

Copyright (C) 2013 Z. Kheirandish et al. This is an open access article distributed under the Creative Commons Attribution License, which permits unrestricted use, distribution, and reproduction in any medium, provided the original work is properly cited.

\begin{abstract}
The present work details a numerical simulation of forced convective laminar flow in a channel with a heated obstacle attached to one wall. The second law analysis is employed to investigate the distribution of entropy generation in the flow domain to demonstrate the rate of irreversibilities in thermal system. The conjugate problem including the convection heat transfer in the fluid flow and conduction one inside the obstacle is solved numerically to obtain the velocity and temperature fields in both gas and solid phases. To reach this goal, the set of governing equations including momentum and energy equations for the gas phase and conduction equation for the obstacle are solved by CFD technique to determine the hydrodynamic and thermal behaviors of the fluid flow around the obstacle and the temperature distribution in the solid element. An attempt is made to detail the local Nusselt number distribution and mean Nusselt number and also the local entropy generation distribution for the individual exposed obstacle faces. A good consistency is found between the present numerical results with experiment.
\end{abstract}

Keywords: Conjugated Heat Transfer; Obstacle; Forced Convection Flow; Entropy Generation

\section{Introduction}

In many thermal systems, convection flow is concerned to procure the precise thermal control. Besides, in heat exchange devices, high performance, light weight and compact heat transfer components are design scope. To achieve these goals, extended surfaces are widely used in heat exchange devices and the design of optimized fins has become increasingly important nowadays. There were numerous studies on fins performance and it has found out that fins increase the rate of convection heat transfer by increasing in fluid mixing and also interrupting the development of thermal boundary layer on the heated surfaces.

Several researchers studied heat transfer enhancement in forced convection duct flow using obstacles with different shapes. Young and Vafai $[1,2]$ focused on special selection of obstacle size and thermal conductivity and found out those significant positive effects of them on the flow and heat transfer characteristics. They numerically studied heated square fin on 2-D laminar flow by finite element method. Also they did an experimental study to find the effect of one, three and five fins on the fluid flow

\footnotetext{
${ }^{*}$ Corresponding author.
}

behavior in a wide range of the Reynolds numbers [3]. Chen and Huang [4] studied position of fins and their arrangement. They investigated force convection cooling of fin arrays in a 2-D channel flow and concluded more mixing in fluid causes an increase in convective heat transfer rate.

Numerical techniques in solving the set of governing equations have special role on results accuracy and required run time. In the related subject, Carvalho et al. [5] did a theoretical study for convection cooling in duct flow. They compared different schemes by analyzing hydraulic behavior of laminar flow in a channel with mounted obstacle and found that quadratic upstream scheme has the most advantageous regarding accuracy against computing time and storage space.

Chen et al. [6] did an experimental research on 3-D channel flow with drop shape fin. It was reported in their paper that the drop shape fin has better thermal performance than circular one.

In another research, Korichi and Oufer [7] studied on channel containing mounted obstacles, in which two obstacles mounted on the lower wall and the last one on the upper wall of a 2-D channel. They investigated a numerical study on laminar convective flow and studied the 
effect of Reynolds number, block spacing and dimensions and also solid to fluid thermal conductivity ratio. Their results showed that increasing in Reynolds number causes more heat removal from the obstacles. Also, it was revealed that maximum heat removal occurred around the obstacle corners.

Li et al. [8] carried out an experiment to investigate the hydrodynamic and thermal behaviors of forced convection flow in rectangular channel with staggered arrays of elliptic and circular fins. Their results showed that the rate of cooling by the elliptic fin is more than that of circular one.

On the other hand, the optimum condition for any process can be determined by the entropy generation analysis because one of the primary objectives in the design of any energy system is to conserve the useful energy applied to take place a certain process. The ireversibilities associated within the process components destroy the useful energy. It is clear that using fins in convection cooling system increases the amount of irreversibilities. Because of the second law of thermodynamics, irreversibility can not be avoided completely but it can be minimized in order to save the available energy. The present work also deals with the second law analysis in convection duct flow with fin to carry out the rate of irreversibilities due to the presence of obstacle.

In the related subject, Bejan [9] obtained a systematic methodology to calculate irreversibility through fluid flow and heat transfer in heat exchangers. Chen et al. [10] studied transverse fin in laminar forced convection channel flow and analyzed entropy generation. They used vorticity stream function method to solve the continuity and momentum equations for fluid flow. They found that fins increase the rates of irreversibilities, both due to viscous effect and irreversible heat transfer process, although they disturb developing of thermal boundary layer which leads to heat transfer enhancement.

In several researches, entropy generation were studied in detail for different flow and channel conditions. Ko et al. [11] carried out a numerical study on wavy channel to investigate entropy generation of laminar forced convection flow ( $R e=100$ up to 400$)$. Their studies showed that for high Reynolds number convection flows, irreversibilities are minimums when duct width to height ratio is equal to unity. In another study, they numerically analyzed entropy generation produced by a forced convective flow in a curved rectangular duct with external heating [12]. Three important factors such as Dean number, external wall heat flux and cross-sectional aspect ratio on entropy generated from frictional irreversibility and heat transfer irreversibility were investigated in detail. It was shown that, at larger Dean number and smaller wall heat flux, frictional irreversibility is the most impressive source of entropy generation; whereas and vice versa, condition for Dean number and wall heat flux, the entropy generation is dominated by heat transfer irreversibility. Also, Ko [13] investigated the effect of longitudinal ribs on laminar forced convection and entropy generation in a curved rectangular duct. He found that the number of mounted ribs and their arrangement have influential effect on flow characteristics and temperature distributions. Ko et al. [14] did a numerical study on entropy generation by turbulent forced convective flow in a curved rectangular duct with various aspect ratios. It was found that the duct aspect ratio has great effect on the distribution of local entropy generation number through the flow domain.

Although there are many studies about numerical analysis of convective cooling in channel and also about the analysis of such thermal systems by computing the entropy generation, a careful inspection of literatures shows that the entropy generation analysis in convective cooling duct flow with obstacles that leads to a conjugate problem is still not studied. Therefore, the present research deals with the investigation of entropy generation in a forced convection flow adjacent to an obstacle in a duct with conjugate problem for the fist time. Toward this end, the set of governing equations consists of the continuity, Navier-Stokes and energy equations for the fluid flow and conduction equation for the obstacle are solved numerically by the CFD method. Because the Cartesian coordinate system is used for this computation, the block off method is employed for simulating the obstacle in the computational domain.

\section{Theory}

Computational domain of the problem is shown in Figure 1. Laminar convective flow enters a 2-D channel which a heated obstacle mounted on bottom wall. Fluid has uniform temperature $T_{i n}$ and parabolic velocity at the inlet of channel. The duct walls are kept insulated except the lower edge of fin which is maintained at constant temperature $T_{w}$ which is more than fluid inlet temperature.

The height of the duct is $H$ and the lengths of the duct upstream and downstream sides of the fin are $L_{i}$ and $L_{e}$, respectively. This is made to ensure that the flows at the inlet and outlet sections are not affected significantly by the sudden change in the geometry and flow at the exit section becomes fully developed. The height and width of the fin are denoted by $L$ and $D$ such that $L=D=0.25$ $H$ is considered in all of the subsequent calculations.

\section{Basic Equations}

The non-dimensional governing equations which are the conservations of mass, $\mathrm{x}$ - and $\mathrm{y}$-momentum and energy for fluid flow in the Cartesian coordinate can be written 


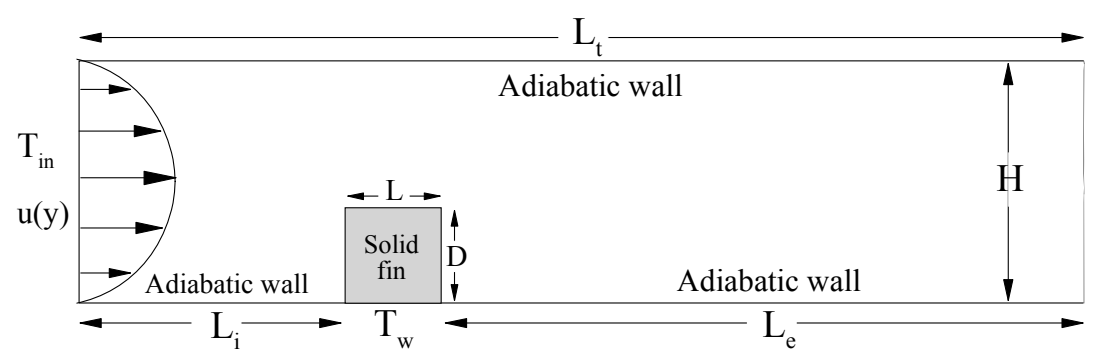

Figure 1. Schematic of the computational domain.

as follows:

$$
\begin{aligned}
& \frac{\partial U}{\partial X}+\frac{\partial V}{\partial Y}=0 \\
& \frac{\partial}{\partial X}\left(U^{2}-\frac{1}{\operatorname{Re}} \frac{\partial U}{\partial X}\right)+\frac{\partial}{\partial Y}\left(U V-\frac{1}{\operatorname{Re}} \frac{\partial U}{\partial Y}\right)=-\frac{\partial P}{\partial X} \\
& \frac{\partial}{\partial X}\left(U V-\frac{1}{\operatorname{Re}} \frac{\partial V}{\partial X}\right)+\frac{\partial}{\partial Y}\left(V^{2}-\frac{1}{\operatorname{Re}} \frac{\partial V}{\partial Y}\right)=-\frac{\partial P}{\partial Y} \\
& \frac{\partial}{\partial X}\left(U \Theta-\frac{1}{P e} \frac{\partial \Theta}{\partial X}\right)+\frac{\partial}{\partial Y}\left(V \Theta-\frac{1}{P e} \frac{\partial \Theta}{\partial Y}\right)=0
\end{aligned}
$$

For solid phase, the conduction equation is considered as follows:

$$
\left(\frac{\partial^{2} \Theta}{\partial X^{2}}\right)+\left(\frac{\partial^{2} \Theta}{\partial Y^{2}}\right)=0
$$

In the above equations, the dimensionless parameters are defined as:

$$
\begin{aligned}
& (X, Y)=\left(\frac{x}{H}, \frac{y}{H}\right),(U, V)=\left(\frac{u}{U_{0}}, \frac{v}{U_{0}}\right), \Theta=\frac{T-T_{i n}}{T_{w}-T_{i n}}, \\
& P=\frac{p}{\rho U_{0}^{2}}, \operatorname{Re}=\frac{U_{0} H}{v}, \operatorname{Pr}=\frac{v}{\alpha}, P e=\operatorname{Re} \cdot \operatorname{Pr}
\end{aligned}
$$

\section{Boundary Conditions}

As mentioned above, fully developed gas flow with parabolic profile and uniform ambient temperature $T_{\text {in }}$ enters the channel. Channel walls are insulated except the obstacle lower wall which is imposed on constant temperature of $T_{w}$. The adiabatic wall condition is selected to elucidate the principal aspects of parametric changes in the heated obstacle to the flow and thermal fields within the channel. On all solid surfaces, no-slip condition is employed for velocity. At the outlet section, zero axial gradients for velocity components and fluid temperature are applied. Finally on the solid-gas interfaces, the continuity of temperature and heat flux is considered that leads to the following equations:

$$
\Theta_{f}=\Theta_{s}
$$

$$
k_{s} \frac{\partial \Theta_{s}}{\partial n}=k_{f} \frac{\partial \Theta_{f}}{\partial n}
$$

The value of local Nusselt number on the heated surfaces can be calculated as follows:

$$
N u=\frac{h_{c} h}{k_{f}}=\frac{1}{\left(\Theta_{s}-\Theta_{m}\right)} \frac{\partial \Theta}{\partial n}
$$

In this formula, $n$ is normal direction to the heated surface and $\Theta_{m}$ is the fluid mean temperature which is determined according to the following equation:

$$
\Theta_{m}=\frac{1}{U H} \int_{H} \Theta \mathrm{d} Y
$$

The mean values of Nusselt number on each obstacle walls can be calculated by [2]:

$$
\overline{N u}_{i}=\frac{\int_{A_{i}} N u_{x} \mathrm{~d} x}{A_{i}}
$$

Subsequently, the value of mean Nusselt number on the obstacle surface which is exposed to the fluid flow is calculated as follow [2]:

$$
N u_{m}=\frac{\sum_{i=l, t, r} \overline{N u}_{i} \cdot A_{i}}{A_{l}+A_{t}+A_{r}}
$$

\section{Entropy Generation}

In the entropy generation analysis, physical quantities of interest are the entropy generation number and Bejan number that can be obtained by the second law analysis. For this purpose the following dimensionless quantities are defined:

$$
\begin{array}{ll}
N S=\frac{s_{g e n}^{\prime \prime} D_{h}^{2}}{k \tau^{2}} & \tau=\frac{T_{w}-T_{i n}}{T_{i n}} \\
B r=\frac{\mu U_{0}^{2}}{k\left(T_{w}-T_{i n}\right)} & \Psi=\frac{B r}{\tau}
\end{array}
$$

In the above equations, $N s$ is the entropy generation number, $S_{\text {gen }}^{\prime \prime}$ the volume rate of entropy generation, $\mathrm{Br}$ the Brinkman number and $\tau$ is the non-dimensional temperature parameter. Using the above parameters, the en- 
tropy generation in dimensionless form can expressed as [15]:

$$
\begin{aligned}
& N s=\left[\left(\frac{\partial \Theta}{\partial X}\right)^{2}+\left(\frac{\partial \Theta}{\partial Y}\right)^{2}\right] \\
& +\Psi\left\{2 \times\left[\left(\frac{\partial U}{\partial X}\right)^{2}+\left(\frac{\partial V}{\partial Y}\right)^{2}\right]+\left[\left(\frac{\partial U}{\partial Y}\right)+\left(\frac{\partial V}{\partial X}\right)\right]^{2}\right\}
\end{aligned}
$$

Above equation contains two parts. The first term on the right represents entropy generation due to the heat transfer:

$$
N s_{\text {cond }}=\left[\left(\frac{\partial \Theta}{\partial X}\right)^{2}+\left(\frac{\partial \Theta}{\partial Y}\right)^{2}\right]
$$

Whereas the second term represents the entropy generation due to the fluid viscous effect:

$$
N s_{\text {visc }}=\Psi\left\{2 \times\left[\left(\frac{\partial U}{\partial X}\right)^{2}+\left(\frac{\partial V}{\partial Y}\right)^{2}\right]+\left[\left(\frac{\partial U}{\partial Y}\right)+\left(\frac{\partial V}{\partial X}\right)\right]^{2}\right\}
$$

In the second law analysis, the Bejan number denotes the relative portion of heat transfer entropy generation to total entropy. Accordingly, this parameter is defined as follows:

$$
B e=\frac{N S_{\text {cond }}}{N S_{\text {cond }}+N S_{\text {visc }}}
$$

Also, integration of the local NS parameter over the entire field of the flow domain gives the total entropy generation which shows the amount of irreversibilities due to the both viscous friction and heat transfer as follows:

$$
N s_{t}=\int_{A} N s(X, Y) \mathrm{d} A
$$

where, $A$ is the area of flow domain.

\section{Numerical Procedure}

Finite difference forms of the partial differential Equations (1)-(5) were obtained by integrating over an elemental cell volume with staggered control volume for $x$ and $y$-velocity components. Other variables of interest were computed at the grid nodes. The discretized forms of the governing equations were numerically solved by the SIMPLE algorithm of Patankar and Spalding [16]. Numerical calculations were performed by writing a computer program in FORTRAN. Numerical solutions are obtained iteratively by the line-by-line method such that iterations are terminated when sum of absolute residuals is less than $10^{-4}$ for each equation. By this numerical strategy, the velocity and temperature distributions in the fluid flow and temperature distribution inside the obstacle can be obtained. After calculation of velocity and temperature fields, Equations (13) and (16) are used to solve for the entropy generation number and Bejan number at each grid point in the flow domain. Then, the total entropy generation through the flow is calculated by Equation (17).

To find the grid independence solution, four different meshes are tested in grid study. For this purpose, a forced convection flow in a duct with an obstacle mounted on the lower wall is simulated along a test case. The values of mean Nusselt number $N u_{m}$ on the obstacle walls are computed and are tabulated in Table $\mathbf{1}$ for different mesh sizes. In this test case, the Reynolds number is equal to 400 with $L=D=0.25 H$ and $K=1000$. As it is seen, the grid size of $450 \times 100$ can be chosen to obtain the grid independent solution, such that the subsequent numerical calculations are made based on this grid size. It should be mentioned that a large concentration of nodes in the region of fin base is employed to ensure the accuracy of numerical computations.

\section{Validation of Numerical Method}

To validate the mathematical model as well as the numerical scheme used in the present study, comparison with relevant theoretical results by other investigators is made along a test case. In this problem, a laminar convection flow over a square obstacle in a duct is analyzed. The fin lower wall is imposed by constant heat flux while the duct's walls are kept insulated. Figure 2 shows the distribution of local Nusselt number along the obstacle surface. It is seen that in the area adjacent to the left root of the fin, a poor heat transfer is found due to the local stagnant flow. The maximum local Nusselt number occurs at the upstream corner of the fin caused by an upsurge in the flow velocity. After the point of $N U_{\max }$, there is a decreasing trend for the convection coefficient which is due to the growth of thermal boundary layer. Near to the upper right corner of the fin, $N U$ increases slightly. The increase in heat transfer area around the corner results in augmented heat transfer. At the rear of the fin, an abrupt drop in convection coefficient takes place due to the separated domain and the recirculation effect. However, Figure 2 shows a good consistency between the present numerical results with theoretical findings in Ref. [1].

Table 1. Grid study results.

\begin{tabular}{cc}
\hline Grid nodes & $N u_{m}$ \\
\hline $200 \times 40$ & 8.38 \\
$330 \times 110$ & 8.46 \\
$450 \times 100$ & 8.69 \\
$500 \times 160$ & 8.70 \\
\hline
\end{tabular}


In a similar test case, the value of average Nusselt number on the obstacle surface is calculated and the variation of this parameter with the Reynolds number is plotted in Figure 3 with comparison to experimental data. This figure shows that there is a slight increase in average Nusselt number with $R e$. This figure also shows a good consistency between the present results with experiment.

\section{Result and Discussion}

In this section, a forced convection air flow over an obstacle in a duct is analyzed for obtaining the hydrody- namic and thermal behaviors of the system. Also, in order to show the rate of irreversibilities in the flow domain, the distributions of entropy generation number at different steady conditions are presented based on the second low analysis. All of the subsequent results are about convection duct flow over a cubic obstacle with $L$ $=D=0.25 H, K=k_{s} / k_{f}=1000$ while $R e$ is varied in the range of $[100-700]$. In order to show the flow pattern in convection duct flow over the obstacle, the streamlines are plotted in Figure 4. It is seen that the streamlines are deflected toward the upper wall of the duct as the flow approaches the obstacle. Figure 4 shows

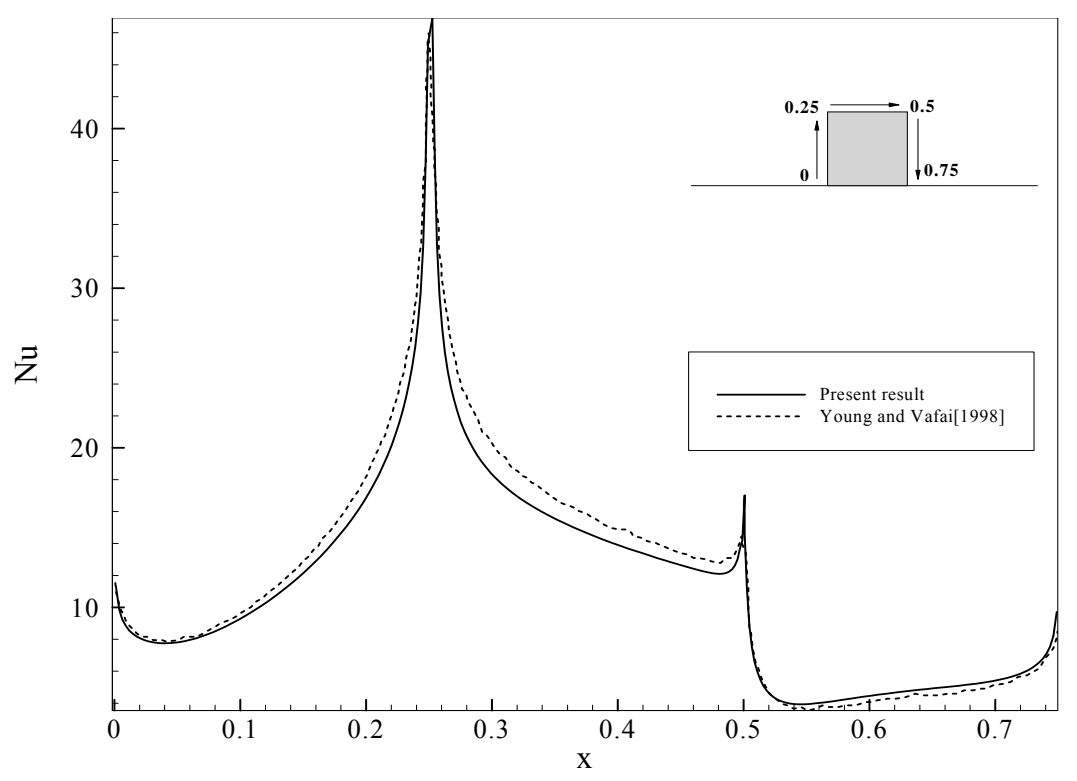

Figure 2. Distribution of Nusselt number along the obstacle walls and comparison with theoretical results by Young and Vafai [1]. $\operatorname{Re}=500, P r=0.72, K=10$ and $L=D=0.25 H$.

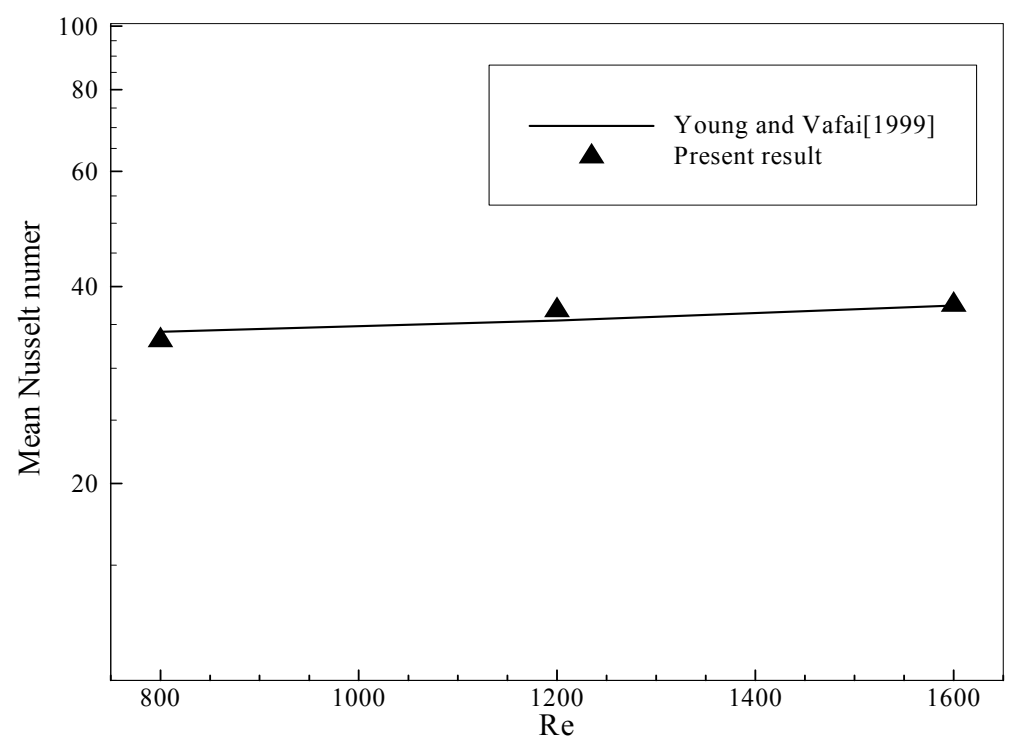

Figure 3. Mean Nusselt number variation with Reynolds number and comparison with experiment $[3] . P r=0.72, K=6818$, $L / H=0.32$ and $D / H=0.29$. 


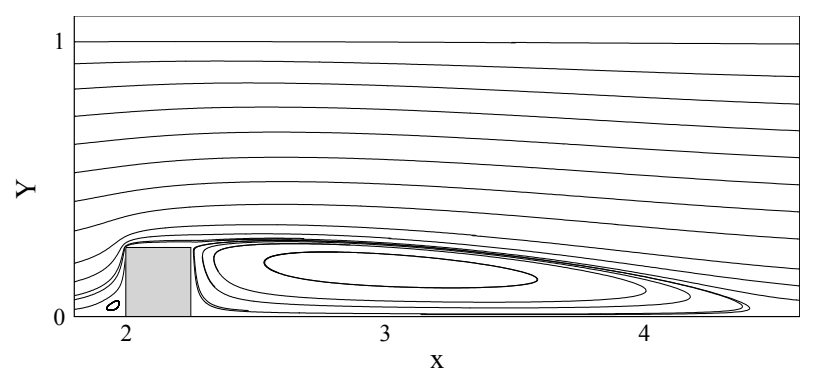

Figure 4. Distribution of streamlines contours, $\boldsymbol{R e}=\mathbf{4 0 0}$.

two recirculated regions adjacent to the fin surface. A very small extent recirculated zone near to the left root of the fin and a large one beyond the protruding fin, which is reattached further downstream of the duct bottom wall.

In Figure 5, the fluid pressure field near to the obstacle is presented by plotting flooded pressure contours in this region. The blocking effect of obstacle in increasing fluid pressure is clearly seen in this figure, such that there is a high pressure domain in upstream side of the obstacle. Also, low pressure regions inside the separated zones near to the obstacle are clearly seen in Figure 5.

The temperature variations in the convection flow and also inside the solid element are shown in Figure 6. It is evident that high temperature region exists near to the heated surface (obstacle bottom wall) and heat removes from this region first by conduction inside the fin and then by convection process in the fluid flow. Because of considering high conductivity ratio $K=k_{s} / k_{f}=1000$ in the computation of Figure 6, the region inside the obstacle becomes nearly as an isotherm one with a temperature which is much closer to the fin base temperature $T_{w}$. The existence of relatively high temperature near to the fin surface and then decreasing in fluid temperature far from the heated obstacle shows how heat removes in this convection flow. In Figure 7, the distributions of local Nusselt number along the obstacle walls for four different values of the Reynolds number are presented. This figure shows that $N u$ has high fluctuations along the heated surfaces of the obstacle such that the maximum value of convection coefficient takes place at the fin upper right corner. This figure shows the same trend as it was observed and explained before in Figure 2, with this fact that the value of convection coefficient on the obstacle surface increases with increasing in Reynolds number.

As it was mentioned before, the amount of irreversibilities (viscous and conductive) at each nodal point inside the thermal system is evaluated in the present paper by the second law analysis and computation of the entropy generation number. The distribution of viscous entropy generation is plotted in Figure 8(a). The maximum value of this parameter takes place near to the upper right corner of the obstacle and the minimum value in the vi- cinity of two stagnant points at the two lower fin corners. From this result, it can be concluded that changing the sharp corner of the fin into a round one can be an effective method in omitting the high reversibility regions from the flow domain. Besides, it is seen that in the region closed to the upper surface of the obstacle, the value of viscous entropy generation is high because the existence of high velocity gradient in this area due to pushing the convection flow by the obstacle toward the upper duct's wall.

The distribution of conductive entropy generation is presented in Figure 8(b). Again it is seen that the maximum entropy generation occurs at the upper right corner of the obstacle. Relatively high rate of irreversibility due to heat transfer takes place in a small region adjacent to the lower duct's wall in upstream side of the fine and in a great extent domain in the rear side of the obstacle. These are the domains in which heat is diffused by conduction at relatively high rate from the heated obstacle toward the convection flow. Besides, Figure 8(b) depicts that the rate of entropy generation by heat transfer is more in the left half of the obstacle domain in comparison to the right half of it. Finally the variation of entropy generation both by heat transfer and viscous effect

$\left(N S=N S_{\text {viscous }}+N S_{\text {conduction }}\right)$ is shown in Figure 8(c). The regions with high rate of irreversibilities can be distinguished by this figure. It is seen that the maximum entropy generation takes place closed to the obstacle surface.

Finally, to study more about the pattern of irreversibility due to both heat transfer and viscous effect in the convection flow, the distribution of Bejan number is plotted in Figure 9. As it was explained before, this parameter shows the relative portion of heat transfer entropy generation to total entropy generation, such that $B e=1$ means that no viscous entropy generation exists in the flow domain and $B e=0$ corresponds to the case in which all of irreversibility is by viscous friction. It is evident that the value of Bejan number must be equal to unity inside the obstacle as it is seen in Figure 9. This figure shows that in the region near to the obstacle and especially at the downstream side of it, the value of Bejan number is relatively high and as we moves far from the obstacle, the value of Bejan number decreases. It means that in the region far from the heated element, viscous effect is the only source of irreversibility.

In the whole domain of any thermal system, total entropy generation which can be calculated by Equation (17) is the only parameter than can show the amount of total irreversibilities take place in the process. Low value of this parameter means that both processes of fluid flow and heat transfer approach to their reversible shapes. The variation of total entropy generation number with $R e$ is plotted in Figure 10. It is seen that $N s_{\text {total }}$ has an increasing 


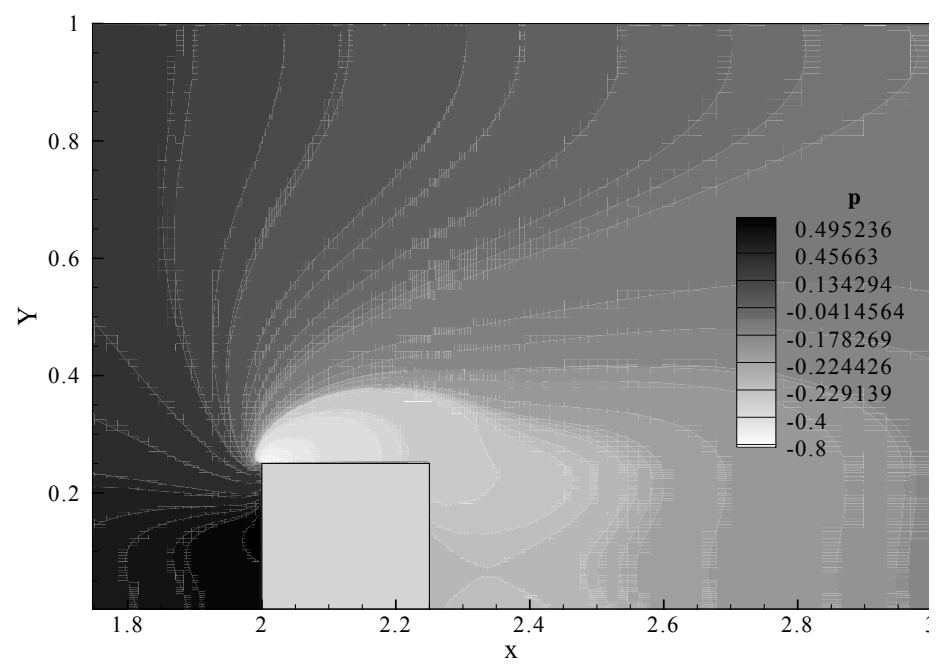

Figure 5. Distribution of pressure near the obstacle, $R e=400$.

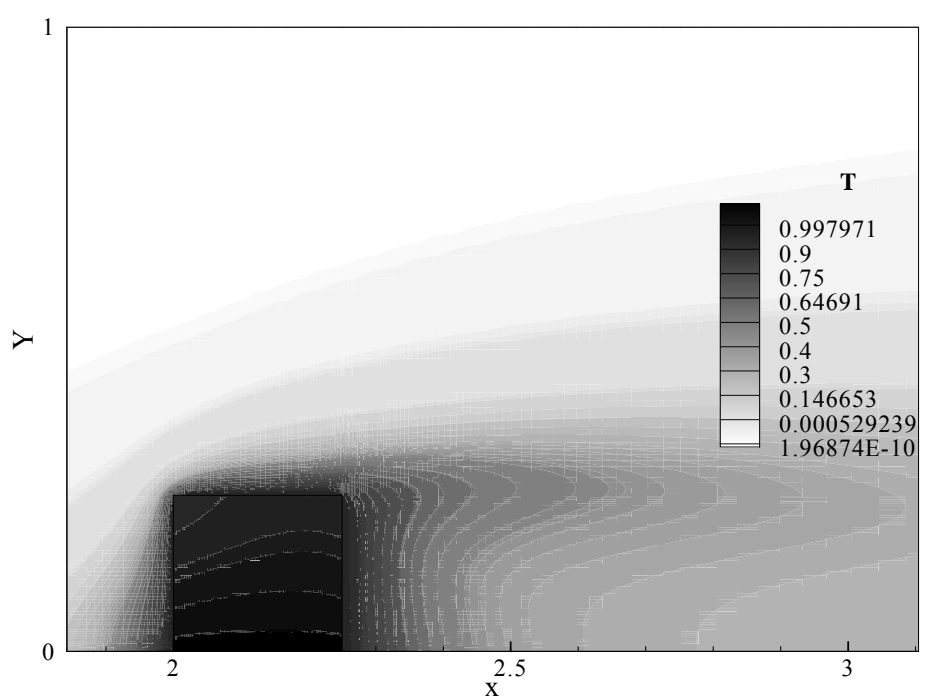

Figure 6. Temperature distribution near the obstacle, $R e=400$.

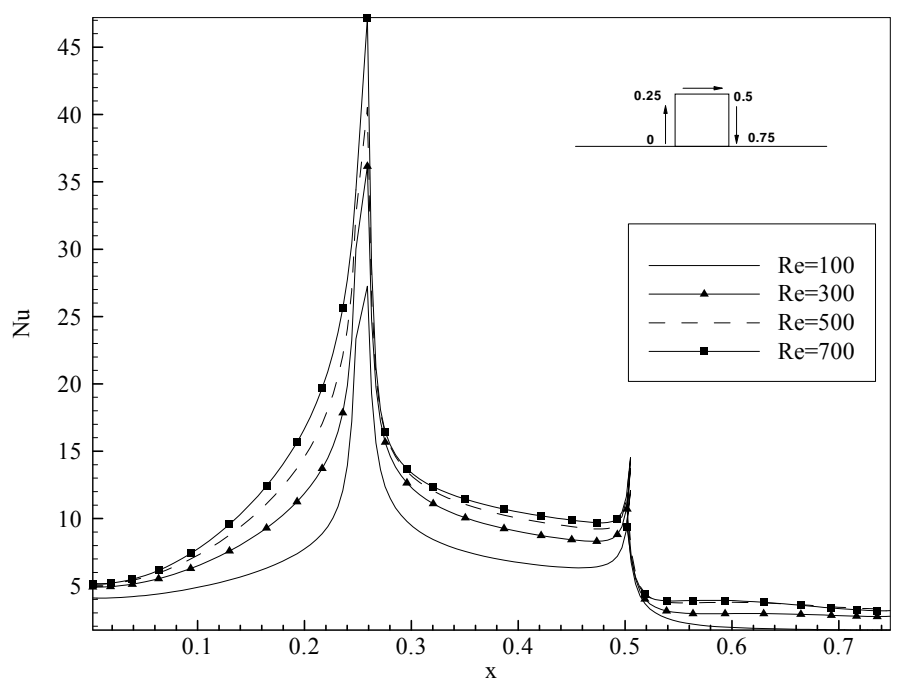

Figure 7. Variation of $\mathrm{Nu}$ on the obstacle walls at different values of the Reynolds number. 


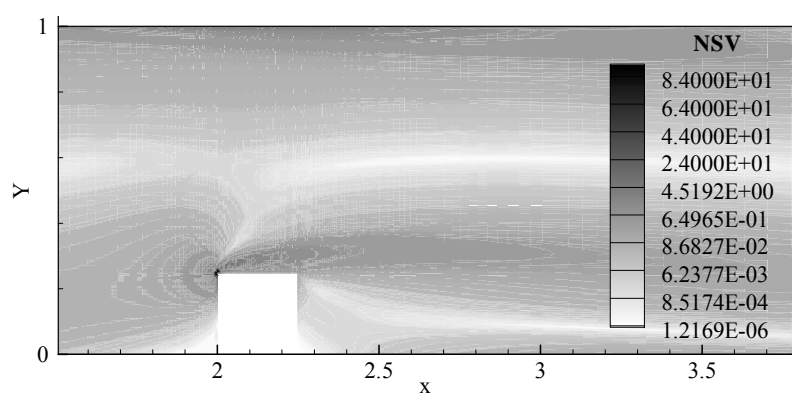

(a)

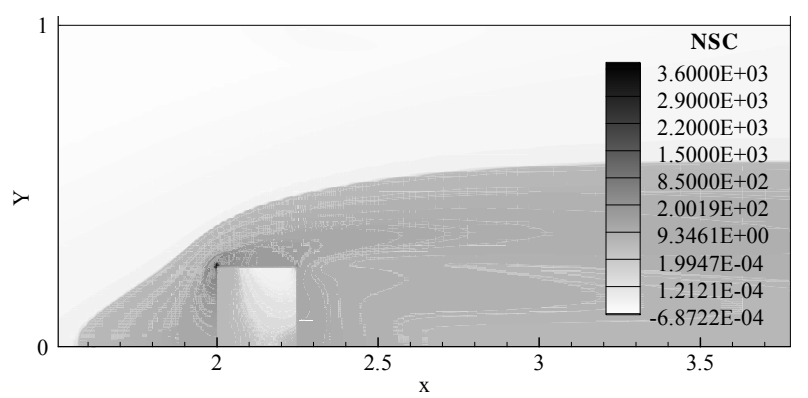

(b)

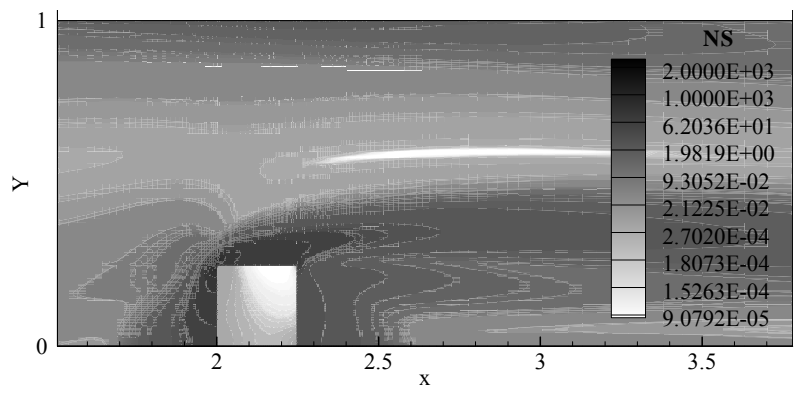

(c)

Figure 8. Distributions of entropy generation numbers in the convection flow near the obstacle, $R e=400$. (a) $N S_{\text {viscous }}$; (b) $N S_{\text {conduction }}$; (c) $N S=N S_{\text {viscous }}+N S_{\text {conduction }}$.

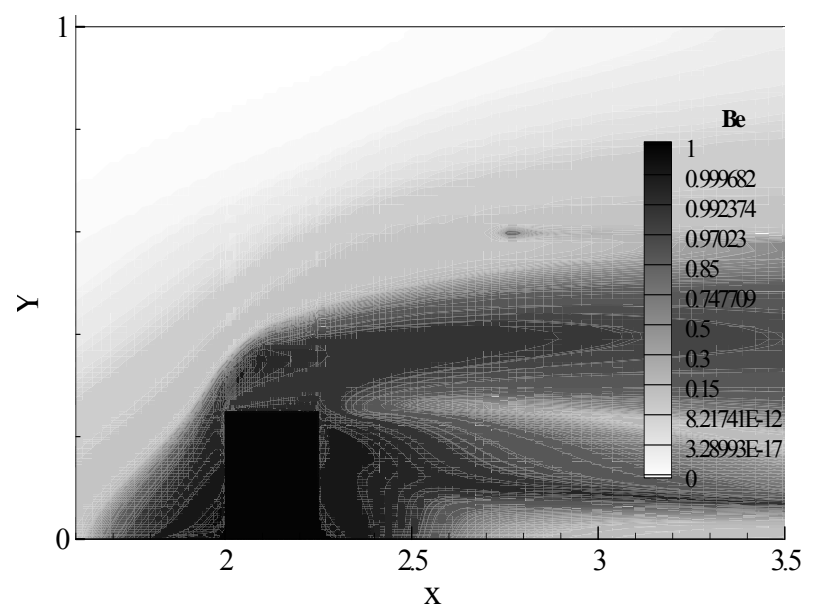

Figure 9. Distribution of Bejan number near the obstacle, $\operatorname{Re}=\mathbf{4 0 0}$.

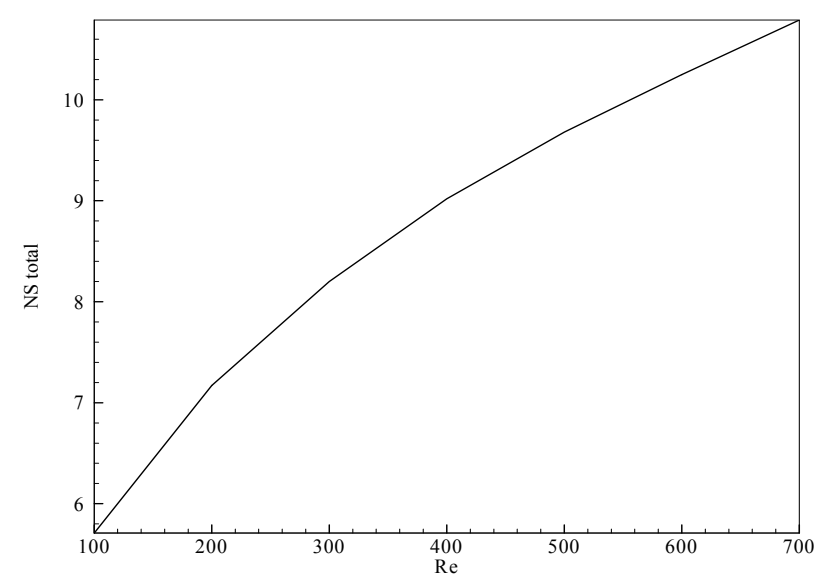

Figure 10. Variation of total entropy generation number with Reynolds number.

trend by increase in $R e$. It means that more irreversibilities take place in high Reynolds number convection flows in comparison to the flows with small $R e$.

\section{Conclusion}

In the present work, second law analysis is done for laminar convection duct flow over a heated obstacle in order to determine the performance of convective cooling. The Navier-Stokes and energy equations for convection flow and conduction equation for the obstacle are solved numerically in a conjugate problem to determine the velocity and temperature distributions. Then, the value of entropy generation number that can show the rate of irreversibility in any thermal system is calculated from the second law of thermodynamics. Numerical results can be very useful in designing such thermal systems with high performance.

\section{REFERENCES}

[1] T. J. Young and K. Vafai, "Convection Cooling of a Heated Obstacle in a Channel," International Journal of Heat and Mass Transfer, Vol. 41, No. 20, 1998, pp. 31313148. doi:10.1016/S0017-9310(97)00323-2

[2] T. J. Young and K. Vafai, "Convective Flow and Heat Transfer in a Channel Containing Multiple Heated Obstacles," International Journal of Heat and Mass Transfer, Vol. 41, No. 21, 1998, pp. 3279-3298. doi:10.1016/S0017-9310(98)00014-3

[3] T. J. Young and K. Vafai, "Experimental and Numerical Investigation of Forced Convective Characteristic of Arrays of Channel Mounted Obstacles," Journal of Heat Transfer, Vol. 121, No. 1, 1999, pp. 34-42.

[4] C. H. Cheng and W. H. Huang, "Numerical Prediction for Laminar Forced Convection in Parallel-Plate Channel with Transverse Fin Array," International Journal of Heat and Mass Transfer, Vol. 34, No. 11, 1991, pp. 27392749. doi:10.1016/0017-9310(91)90232-4 
[5] M. G. Carvalho, F. Durst and J. C. F. Pereira, "Prediction and Measurements of Laminar over Two-Dimensional Obstacles," Applied Mathematical Modelling, Vol. 11, No. 1, 1987, pp. 23-34.

[6] Z. Chen, Q. Li, D. Meier and H. J. Warnecke, "Convective Heat Transfer and Pressure Loss in Rectangular Duct with Drop-Shaped Pin Fins," Heat and Mass Transfer, Vol. 33, No. 3, 1997, pp. 219-224. doi:10.1007/s002310050181

[7] A. Korichi and L. Oufer, "Numerical Heat Transfer in a Rectangular Channel with Mounted Obstacles on Upper and Lower Walls," International Journal of Thermal Sciences, Vol. 44, No. 7, 2005, pp. 644-655. doi:10.1016/j.ijthermalsci.2004.12.003

[8] Q. Li, Z. Chen, U. Flechtner and H. J. Warnecke, "Heat Transfer and Pressure Drop Characteristics in Rectangular Channel with Elliptic Fins," International Journal of Heat and Fluid Flow, Vol. 19, No. 3, 1998, pp. 245-250. doi:10.1016/S0142-727X(98)00003-4

[9] A. Bejan, "Entropy Generation through Heat and Fluid Flow," Wiley, New York, 1982.

[10] C. H. Cheng and W. H. Huang, "Entropy Generation and Heat Transfer via Laminar Forced-Convection Channel Flow over Transverse Fins in Entrance Regions," Applied Energy, Vol. 32, No. 4, 1989, pp. 241-267. doi:10.1016/0306-2619(89)90015-9

[11] T. H. Ko and C. S. Cheng, "Numerical Investigation on Developing Laminar Forced Convection and Entropy Generation in a Wavy Channel," International Commu- nications in Heat and Mass Transfer, Vol. 34, No. 8, 2007, pp. 924-933.

doi:10.1016/j.icheatmasstransfer.2007.05.021

[12] T. H. Ko and K. Ting, "Entropy Generation and Optimal Analysis for Laminar Forced Convection in Curved Rectangular Ducts: A Numerical Study," International Communications in Heat and Mass Transfer, Vol. 45, No. 2, 2006, pp. 138-150.

[13] T. H. Ko, "A Numerical Study on Entropy Generation and Optimization for Laminar Forced Convection in a Rectangular Curved Duct with Longitudinal Ribs," International Communications in Heat and Mass Transfer, Vol. 45, No. 11, 2006, pp. 1113-1125.

[14] T. H. Ko and C. P. Wu, "A Numerical Study on Entropy Generation Induced by Turbulent Forced Convection in a Curved Rectangular Duct with Various Aspect Ratios," International Communications in Heat and Mass Transfer, Vol. 36, No. 1, 2009, pp. 25-31. doi:10.1016/j.icheatmasstransfer.2008.08.016

[15] E. Abu-Nada, "Investigation of Entropy Generation over a Backward Facing Step under Bleeding Conditions," Energy Conservation and Management, Vol. 49, No. 11, 2008, pp. 3237-3242. doi:10.1016/j.enconman.2007.10.031

[16] S. V. Patankar and D. B. Spalding, "A Calculation Procedure for Heat, Mass and Momentum Transfer in ThreeDimensional Parabolic Flows," International Journal of Heat and Mass Transfer, Vol. 15, No. 10, 1972, pp. 17871806. doi:10.1016/0017-9310(72)90054-3 


\section{Nomenclature}

$B e$ : Bejan number

$B r$ : Brinkman number

$D$ : obstacle height (m)

$D_{h}$ : hydraulic diameter $(2 H)$

$h$ : height of channel

$h_{c}$ : convection heat transfer coefficient

$H$ : height of the channel $(\mathrm{m})$

$k$ : thermal conductivity $\left(\mathrm{W} / \mathrm{m} \cdot{ }^{\circ} \mathrm{C}\right)$

$K$ : thermal conductivity ratio $\left(\frac{k_{s}}{k_{f}}\right)$

$L$ : obstacle length (m)

$L t$ : total length of the duct (m)

$N s$ : entropy generation number

$\mathrm{Nu}$ : Nusselt number

$p$ : pressure $(\mathrm{Pa})$

$P$ : dimensionless pressure

$P e$ : Peclet number

Pr: Prandtl number

$s_{g e n}^{\prime \prime}$ : volume rate of entropy generation $\left(\mathrm{W} / \mathrm{m}^{3} \cdot \mathrm{K}\right)$

$T$ : temperature $(\mathrm{K})$

$u$ : $\mathrm{x}$-velocity component $(\mathrm{m} / \mathrm{s})$

$U$ : dimensionless $\mathrm{x}$-velocity component

$U_{0}$ : mean velocity at the inlet section

$v$ : y-velocity component $(\mathrm{m} / \mathrm{s})$
$V$ : dimensionless y-velocity component

$x$ : horizontal coordinate $(\mathrm{m})$

$X$ : dimensionless form of $x$

$y$ : vertical coordinate $(\mathrm{m})$

$Y$ : dimensionless form of $y$

\section{Greek Symbols}

$\alpha:$ thermal diffusivity $\left(\mathrm{m}^{2} / \mathrm{s}\right)$

$\Theta$ : dimensionless temperature

$\mu$ : dynamic viscosity $\left(\mathrm{N} \cdot \mathrm{s} / \mathrm{m}^{2}\right)$

$v$ : kinematic viscosity $\left(\mathrm{m}^{2} / \mathrm{s}\right)$

$\rho: \operatorname{density}\left(\mathrm{kg} / \mathrm{m}^{3}\right)$

$\tau:$ dimensionless temperature parameter

$\Psi$ : viscous dissipation number

\section{Subscripts}

cond : conduction

$e$ : end

$f$ : fluid

in: inlet section

$s:$ solid

$t$ : total

visc: viscous

$w$ : wall 